\title{
Efecto del aula virtual en el aprendizaje de la asignatura de administración general
}

\author{
The effect of a virtual classroom in the learning process of a general management course
}

\author{
José Alcántara Casquier'; Juan Dueñas Béjar²; María E. Villanueva Pinedo ${ }^{3}$
}

\begin{abstract}
Resumen
En el marco del proceso de enseñanza y aprendizaje nos encontramos con varios problemas, uno de ellos es la falta de motivación en los estudiantes para el aprendizaje significativo. Este trabajo tuvo como objetivo general determinar el efecto que produce el aula virtual (AV) en el aprendizaje de la asignatura de Administración General (AG) en los estudiantes de la Universidad Nacional Agraria La Molina (UNALM), cuando se usa como recurso didáctico complementario en la enseñanza. El objetivo complementario fue determinar la satisfacción de los estudiantes cuando se utiliza como recurso didáctico el AV en la enseñanza de la asignatura AG. Para ello, se utilizó un diseño cuasi-experimental con grupos de control $(n=34)$ y experimental $(n=34)$ con la aplicación de pre y post test. Los resultados muestran que los estudiantes que accedieron al $\mathrm{AV}$, grupo experimental, tuvieron en promedio un mejor rendimiento que los estudiantes que no usaron el AV, grupo control. En conclusión, el AV como complemento del aprendizaje permite que los estudiantes mejoren su rendimiento.
\end{abstract}

Palabras clave: enseñanza-aprendizaje; administración en línea; planificación; Matemáticas para administración.

\begin{abstract}
In the framework of the teaching and learning process we are faced with several problems, one of them is the lack of motivation in students for meaningful learning. This work had as a general goal, to determine the effect that produces the Virtual Classroom (AV) on the learning of the subject of the General Administration (GA) course, in the students of the Universidad Nacional Agraria La Molina (UNALM), when used as supplementary teaching resource in education. As a supplementary goal, the idea was to determine the satisfaction that students had when they used the AV as a supplementary teaching resource of the AG discipline. For this purpose we used a quasi-experimental design with control groups $(\mathrm{n}=34)$ and experimental $(\mathrm{n}=34)$ applying pre and post-tests. The results showed that students who agreed to use the AV, (experimental group), had a better performance than the students who did not use the AV, (control group). In conclusion, the $\mathrm{AV}$ as a supplement to learning, allows students to improve their performance.
\end{abstract}

Keywords: teaching-learning process; on-line administration; planning; Mathematics for administration.

\section{Introducción}

Existen numerosos trabajos presentados en diferentes actividades desarrolladas sobre educación donde se insiste en que la computadora es un recurso que debe ser incorporado para presentar los conocimientos, para permitir que los estudiantes realicen algunas pruebas de lo que están aprendiendo o como un complemento para su aprendizaje.

En actualidad, las TIC nos brindan una serie de herramientas que permiten realizar presentaciones dinámicas de acuerdo con lo que nosotros queremos mostrar y de esta manera presentar los nuevos conocimientos de diferentes formas.
A la vez, esto contribuye a que los conocimientos permanezcan por un tiempo más prolongado que el de una clase presencial y el estudiante pueda volver a revisarlos afianzando su aprendizaje y favoreciendo, además, los diferentes estilos de aprendizaje de los alumnos.

Barroso (2007) expresa que "Gran parte del desarrollo de las modernas técnicas educativas se basa en el hecho que cuantos más sentidos participen en el proceso de aprendizaje, más fácil será la asimilación y retención de conocimientos". Asimismo, el enfoque cognoscitivo y significativo de David Ausubel, el aprendizaje representativo y por descubrimiento de Jérome Bruner,

\footnotetext{
1 Docente adscrito a la Maestría en Nutrición Pública. Universidad Nacional Agraria La Molina, Lima, Perú. Email: jalcantara@lamolina.edu.pe

2 Profesor del Departamento Académico de Matemática, Facultad de Ciencias. Universidad Nacional Agraria La Molina, Lima, Perú. Email: duenas@lamolina.edu.pe

3 Profesor del Departamento Académico de Matemática, Facultad de Ciencias. Universidad Nacional Agraria La Molina, Lima, Perú. Email: villanuepi@lamolina.edu.pe
} 
el enfoque del procesamiento de información de Robert Gagné, el enfoque evolutivo de Jean Piaget y el enfoque histórico cultural de Lev Vigotsky, son los marcos teóricos contemporáneos que ayudan a entender mejor el aprendizaje humano y, en especial, el aprendizaje significativo. Ausubel (1983) afirma que "La esencia del proceso del aprendizaje significativo reside en que ideas expresadas simbólicamente son relacionadas de modo no arbitrario y sustancial (no al pie de la letra) con lo que el alumno ya sabe." y que: "El aprendizaje significativo presupone tanto que el alumno manifieste una actitud de aprendizaje significativo; es decir, una disposición para relacionar sustancial y no arbitrariamente el nuevo material con su estructura cognoscitiva, como que el material que aprende es potencialmente significativo para él, es decir, relacionable con su estructura de conocimiento sobre una base no arbitraria y no al pie de la letra." Ausubel (1983) acuña la expresión Aprendizaje Significativo para contrastarla con el Aprendizaje Memorístico. Él afirma que las características del Aprendizaje Significativo son: Los nuevos conocimientos se incorporan en forma sustantiva en la estructura cognitiva del estudiante y esto se logra gracias a su esfuerzo deliberado por relacionar los nuevos conocimientos con sus conocimientos previos, producto de una implicación afectiva del estudiante; es decir, este quiere aprender aquello que se le presenta porque lo considera valioso. En cambio, el Aprendizaje Memorístico se caracteriza porque en el estudiante los nuevos conocimientos se incorporan en forma arbitraria en su estructura cognitiva. Además, no realiza un esfuerzo para integrar los nuevos conocimientos con sus conocimientos previos $\mathrm{y}$, al no haber una implicación afectiva del estudiante, porque no concede valor a los contenidos presentados por el profesor, no quiere, realmente, aprender. Castañeda (2002) afirma que es necesario el uso de las nuevas tecnologías de la información y la comunicación porque son parte de un proceso cultural generado a partir de los cambios que van ocurriendo. Entonces, se genera un nuevo paradigma en la educación donde el profesor se convierte en un productor de medios de enseñanza, facilitador de los recursos de aprendizaje y gestionador de aprendizajes personalizados.

Según Horton (2000) "El aula virtual es el medio en la World Wide Web (WWW), en el cual los educadores y educandos se encuentran para realizar actividades que conducen al aprendizaje." y añade que "El aula virtual no debe ser solo un mecanismo para la distribución de la información, sino que debe ser un sistema adonde las actividades involucradas en el proceso de aprendizaje puedan tomar lugar, es decir, que deben permitir interactividad, comunicación, aplicación de los conocimientos, evaluación y manejo de la clase. Las aulas virtuales hoy toman distintas formas y medidas y hasta son llamadas con distintos nombres."

Santoveña (2004) menciona que un entorno virtual flexible será aquel que pueda adaptarse a las necesidades de los estudiantes, que cuente con una interfaz familiar y que presente funcionalidad fácilmente reconocible, que sea sencillo de utilizar y que ofrezca una navegabilidad clara y homogénea en todas sus secciones.

El problema formulado fue ¿Cuál es el efecto del Aula Virtual en el aprendizaje de la asignatura de Administración
General, de los estudiantes de la UNALM, cuando se utiliza como recurso para complementar el proceso de enseñanza-aprendizaje?.

Este trabajo tiene como objetivo general determinar el efecto que produce el Aula Virtual (AV) en el aprendizaje de la asignatura de Administración General (AG) en los estudiantes de la Universidad Nacional Agraria La Molina (UNALM), cuando se usa como recurso didáctico complementario en la enseñanza. Afianzando este objetivo, se propone determinar la satisfacción que produce en los estudiantes el uso del AV en la enseñanza de la asignatura de AG.

\section{Materiales y métodos}

La elaboración de las actividades y los materiales del AV debe darse a partir de la realidad del curso; para ello, se debe planificar lo que se va a preparar y es oportuno considerar una evaluación posterior del material que servirá como retroalimentación del proceso de enseñanza aprendizaje. Con respecto a la fase de diseño y planificación, es importante establecer cuáles son los objetivos de aprendizaje, saber qué contenidos los estudiantes deberán adquirir durante la formación, conocer bien el inicio del aprendizaje, quiénes son los estudiantes y qué nivel de conocimientos tienen, saber con qué recursos se cuenta, decidir y comunicar a nuestros estudiantes cómo evaluaremos su progreso. La evaluación es un elemento fundamental de cualquier entorno formativo y lo es más aún en el Aula Virtual, si se busca aprovecharla para mejorar el aprendizaje (Cedrían, 2009 y Bautista, 2008). El medio que se ha usado fue el Aula Virtual (AV), con Plataforma Moodle de la UNALM y el trabajo se desarrolló de la siguiente manera: Recopilación y organización de la información, diseño del $\mathrm{AV}$, elaboración de cada una de las secciones que conformarían el $\mathrm{AV}$, presentación de esta para su uso y, finalmente, la evaluación en línea de los conocimientos adquiridos y la satisfacción del usuario. El diseño del estudio es cuasi-experimental, con dos grupos apareados, uno experimental (EXP) y otro de control (CONT), a los cuales se les aplicó el pre test (PRE) y post test (POST) como prueba de entrada y salida, los cuales fueron validados a través una prueba piloto. Además, al grupo experimental se le aplicó una encuesta para identificar su satisfacción.

El estudió consideró a la población de 277 estudiantes matriculados en la asignatura de Administración General, en el ciclo 2011-I, a través de una muestra conformada por $n=68$ estudiantes, con grupos experimental $(n=34)$ y control $(n=34)$, que corresponden al $24,5 \%$ del total.

El AV está compuesto de las siguientes secciones: una primera, de inicio, donde se presentará el índice y los objetivos; una segunda, que corresponde a la introducción, donde aparece la prueba de entrada; una tercera, cuarta, quinta, sexta y séptima que contienen el desarrollo teórico detallado, los ejemplos y las referencias bibliográficas; la octava que considera la herramientas matemáticas con un grupo de ejercicios y problemas desarrollados, paso a paso, y otras actividades que permitan el afianzamiento del conocimiento en esta área $\mathrm{y}$, por último, una sección en la que se encuentran las actividades para verificar los logros en el dominio del tema, el examen de salida y la encuesta 


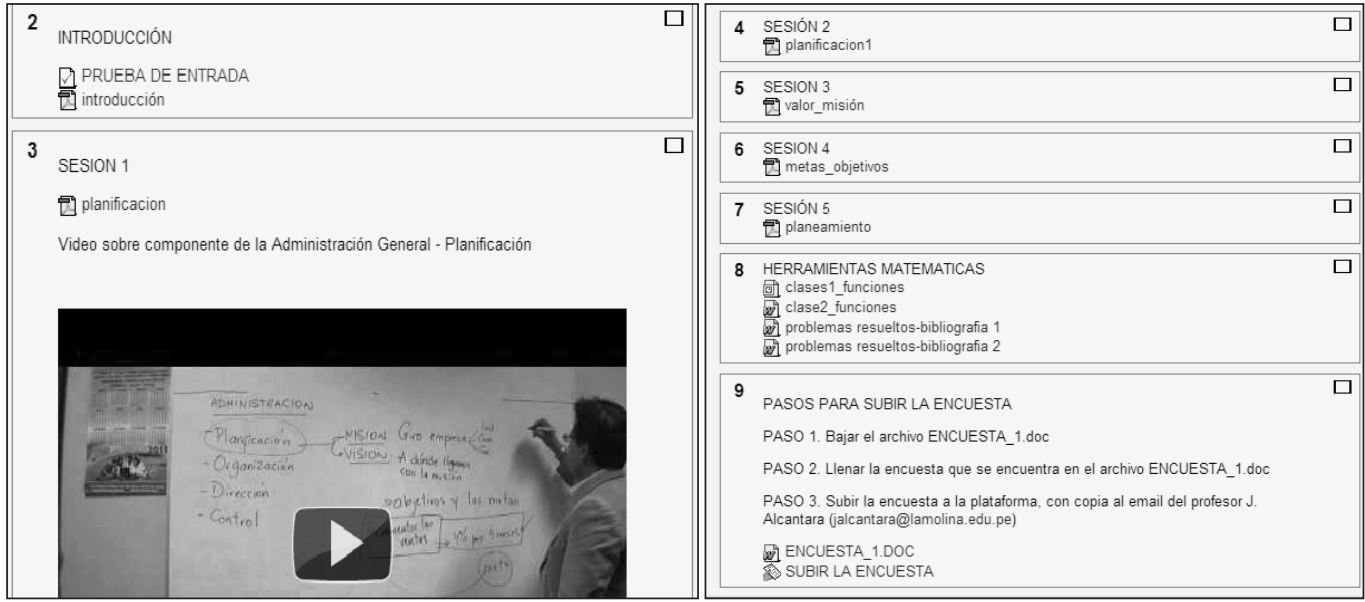

Figura 1. Algunas secciones del aula virtual

para analizar la satisfacción del usuario. En la Fig. 1, se muestran algunas secciones del aula virtual implementadas para la asignatura de Administración General. Para la recolección de datos de aplicaron dos pruebas (pre y post test) para medir los aprendizajes, conformadas por 12 ítems y una encuesta para medir la satisfacción del estudiante con respecto al $\mathrm{AV}$, conformada por ocho ítems, la cual fue validada por tres profesores de Gestión Empresarial.

Usando el software MiniTab versión 14, se realizó el siguiente análisis de los datos: 1) normalidad 2) Prueba Homogeneidad de Varianzas, para elegir el método estadístico adecuado, y para el cumplimiento del objetivo general se utilizó la técnica estadística No Paramétrica de Mann - Whitney. Para el objetivo complementario, se utilizó como instrumento de medida una encuesta.

\section{Resultados y discusión}

Los resultados se presentan a continuación:

En la Tabla 1, se presenta la estadística descriptiva del pre-test de los grupos experimental y control; de los datos obtenidos podemos manifestar que la nota promedio de la prueba de entrada del grupo experimental fluctúa entre $10,244 \pm 2,783$ puntos, siendo la nota mínima de 5,0 y un máximo de 16,7. En cambio, la nota promedio del grupo de control fluctúa entre de 9,943 $\pm 2,923$ puntos, siendo la nota mínima 3,3 y la máxima 16,7 .

Tabla 1. Estadística Descriptiva de la Prueba de Entrada (pre - test)

\begin{tabular}{|c|c|c|}
\hline $\begin{array}{l}\text { Estadístico Grupo } \\
\text { (EXP) }\end{array}$ & Experimental & $\begin{array}{l}\text { Grupo Control } \\
\text { (CONT) }\end{array}$ \\
\hline Media & 10,24 & 9,95 \\
\hline Mediana & 10,00 & 10,00 \\
\hline Moda & 10,00 & 8,30 \\
\hline Desv. Estándar & 2,78 & 2,92 \\
\hline Varianza & 7,75 & 8,55 \\
\hline Rango & 11,70 & 13,40 \\
\hline Mínimo & 5,00 & 3,30 \\
\hline Máximo & 16,70 & 16,70 \\
\hline
\end{tabular}

Tabla 2. Prueba de Anderson-Darling para una muestra

\begin{tabular}{lllll}
\hline & ANTESEXP & DESPEXP & ANTCONT & DESPCONT \\
\hline P_valor & 0,061 & $<0,005$ & 0,077 & $<0,005$
\end{tabular}

En la Tabla 2, se presenta los p-valor para el grupo experimental en el pre-test (ANTESEXP) y en el pos-test (DESPEXP), así como también del grupo control en el pre-test (ANTCONT) y en el post-test (DESPCONT) y se concluye que los puntajes en más de uno de los grupos no se ajustan a una distribución normal, por lo que se emplearán pruebas no paramétricas para comparar puntajes entre grupos diferentes (Prueba de Mann-Whitney) y para comparar puntajes en un mismo grupo (Prueba de Wilcoxon).

De la Tabla 3, se tiene que la significancia de los puntajes del pre-test entre los grupos de control (contpre) y experimental (exppre) no difieren significativamente en cuanto a su nivel, por lo que se puede afirmar que al inicio del experimento ambos grupos tienen puntajes similares. La Fig. 2 corrobora esta afirmación.

En la Tabla 4, se observa la significancia y se concluye que los puntajes del post test del grupo experimental son superiores a los puntajes del pre test en el grupo experimental, lo que muestra la efectividad del uso del AV.

En la Tabla 5, se observa la significancia y se concluye que los puntajes del post-test del grupo experimental son superiores a los puntajes del post-test del grupo control, lo que evidencia la efectividad del AV como complemento del aprendizaje con respecto a la enseñanza tradicional. Por lo tanto, con la afirmación anterior, queda contrastada la hipótesis: "El aula virtual como complemento del aprendizaje de la asignatura de Administración General mejora el rendimiento académico, comparada con la metodología tradicional, de los estudiantes de la UNALM". La Fig. 3 valida esta afirmación.

En la Tabla 6, se observa que con respecto al ítem 1: ¿Utilizó el material en línea sobre el tema de Planificación?, se obtuvo que el 97,67 \% confirmó que sí lo utilizó. En cuanto al ítem 2: ¿El material presentado le ha ayudado a entender mejor el tema?, se tiene que el 97,67 \% estaba de 
Tabla 2. Prueba de comparación entre los puntajes del pre-test de los grupos experimental y control

Prueba de Mann-Whitney e IC: exppre, contpre

$\mathrm{N}$ Mediana

exppre $34 \quad 10,000$

contpre $34 \quad 10,000$

La estimación del punto para ETA1-ETA2 es -0,00095. 1 El porcentaje IC para ETA1-ETA2 es (-1,599 -

$1,699)$

$\mathrm{W}=1193,0$

Prueba de ETA1 $=$ ETA2 vs. ETA1 no es $=$ ETA2 es significativa en 0,8110

La prueba es significativa en 0,8077 (ajustado por empates)

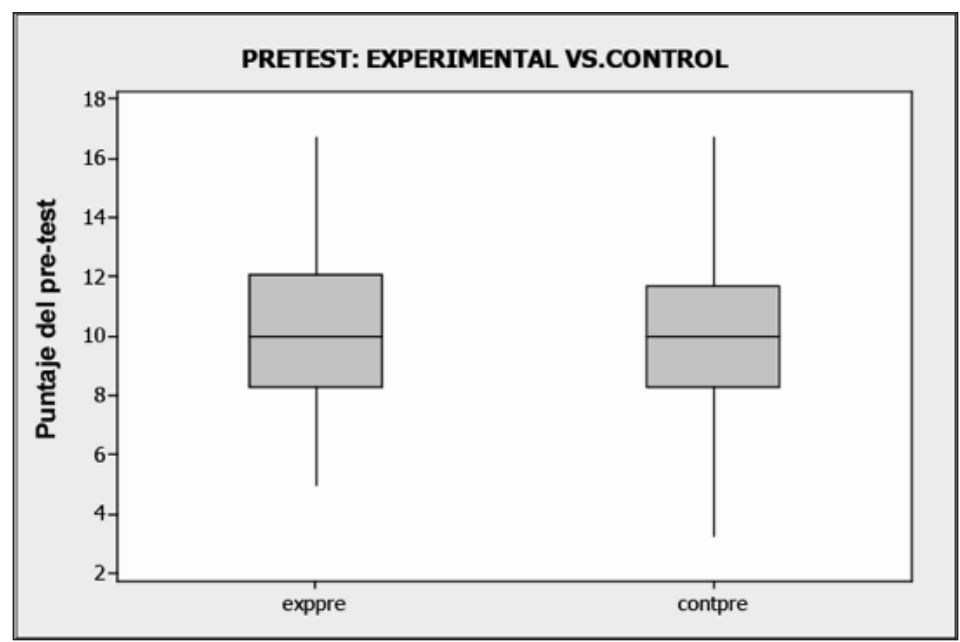

Figura 2. Comparación de los grupos en el pre-test.

Tabla 3. Prueba de comparación entre los puntajes del pre test y post test del grupo experimental Prueba de clasificación con signos de Wilcoxon: difexp

Prueba de la mediana $=0,000000$ vs. la mediana $>0,000000$

Número

de Estadística $\quad$ Mediana

N prueba de Wilcoxon Pval estimada

$\begin{array}{lllll}\text { difexp } 34 \quad 34 & 571,0 & 0,000 & 5,150\end{array}$

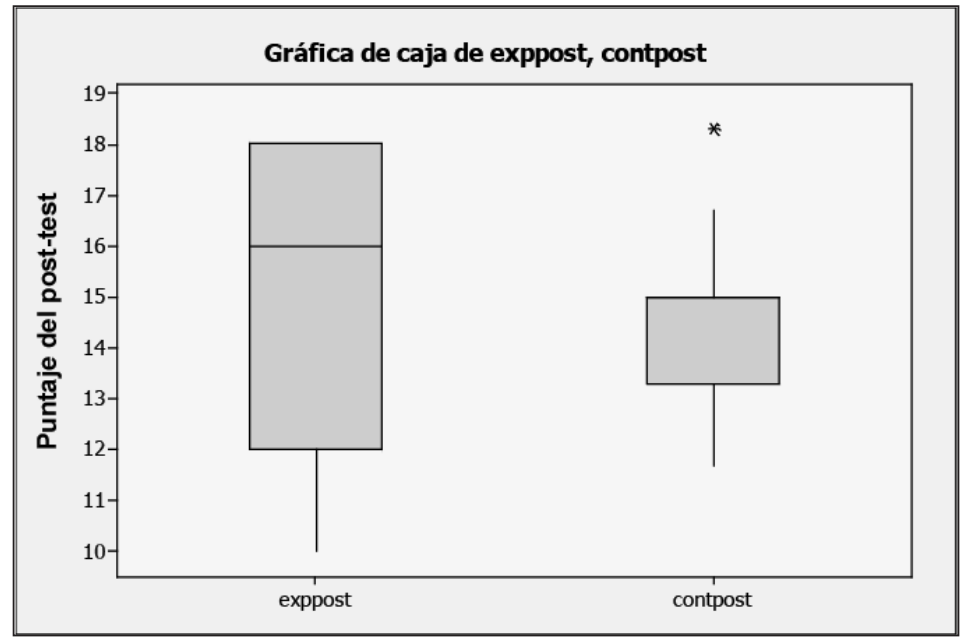

Figura 3. Comparación de grupos en el post test. 
Tabla 4. Cuadro de consolidado (porcentual) de los resultados de la encuesta de satisfacción del estudiante

\begin{tabular}{llrrrrrr}
\hline & & Item1 & Item2 & Item3 & Item4 & Item5 & Item6 \\
\hline \multirow{2}{*}{ Respuestas } & $\mathrm{Si}$ & 97,67 & 97,67 & 95,35 & 69,77 & 88,37 & 32,56 \\
\cline { 2 - 8 } & No & 2,33 & 2,33 & 4,65 & 30,23 & 11,63 & 67,44 \\
\hline
\end{tabular}

acuerdo con que el material le había ayudado a entender mejor el tema. Con relación al ítem 3: ¿Considera que existe un orden en la presentación del contenido?, se tiene que el $95,35 \%$ manifestó que sí existía un orden en la presentación del contenido. Con respecto al ítem 4: ¿Fue sencillo acceder al material en línea?, el 69,77 \% de los encuestados afirmó que sí era sencillo hacerlo. En cuanto al ítem 5: ¿Le pareció adecuada la presentación del material?, el 88,37\% mencionó que sí le pareció adecuada. Con respecto al ítem 6: ¿Hubiese preferido otro diseño para el material?, el 67,44 \% afirmó que no hubiesen preferido un diseño diferente para el material.

\section{Conclusiones}

El uso de un Aula Virtual (AV) no necesariamente tiene que ser independiente de un programa tradicional de curso, sino que puede ser considerado dentro del desarrollo de este y como parte de su diseño instruccional en donde se presenta como un elemento motivador y de apoyo, en este caso, para la asignatura de Administración General (AG). Entonces, podría ser considerado como material didáctico complementario aplicado de esta forma pero también podría ser considerado como recurso didáctico si se desea emplear en el proceso de enseñanza aprendizaje a distancia. De acuerdo con los resultados de pre test aplicado al grupo experimental y control, se tiene que se inicia el trabajo con grupos similares en su rendimiento. Comparando puntajes obtenidos del pre test y post test, se tiene que la aplicación del AV mejora significativamente el proceso de enseñanzaaprendizaje de la asignatura de Administración General en los estudiantes de la UNALM y con respecto a la encuesta los resultados muestran que el AV ha tenido buena acogida por parte de estos.

\section{Literatura citada}

Ausubel, D. y Hanesian, H. 1983. Psicología Educativa: Un punto de vista cognoscitivo. México: Trillas.

Barroso, J. y Romero, R. 2007. La informática, los multimedia y los hipertextos en la enseñanza. En J. Cabero (coord.): Nuevas Tecnologías Aplicadas a la Educación. Madrid, España: McGRAW-HILL, pp. 151-168.

Castañeda, A. 2002. Aplicaciones de las Nuevas Tecnologías de la Informática y las Comunicaciones (NTIC) en el proceso de enseñanza-aprendizaje. Lima, Perú: UPC.

Horton, W. 2000. Designing web based training. Wiley Computer Publisher, New York,

N. Disponible en http://students.ed.uiuc.edu/scagnoli/ pubs/AulaVirtual.pdf

Santoveña, S. 2004. Metodología Didáctica en Entornos Virtuales de Aprendizaje. Disponible en http://www. ugr.es/ sevimeco/revistaeticanet/Numero3/Articulos/ Formateados/metodologia_didactica.pdf 